\title{
A Simplified Statistical Model for the Geomagnetic Field and the Detection of Shallow Bias in Paleomagnetic Inclinations: Was the Ancient Magnetic Field Dipolar?
}

\author{
Lisa Tauxe \\ Scripps Institution of Oceanography, La Jolla, California \\ Dennis V. Kent \\ Department of Geological Sciences, Rutgers University, Piscataway, New Jersey and \\ Lamont-Doherty Earth Observatory, Palisades, New York
}

The assumption that the time-averaged geomagnetic field closely approximates that of a geocentric axial dipole (GAD) is valid for at least the last 5 million years and most paleomagnetic studies make this implicit assumption. Inclination anomalies observed in several recent studies have called the essential GAD nature of the ancient geomagnetic field into question, calling on large (up to $20 \%$ ) contributions of the axial octupolar term to the geocentric axial dipole in the spherical harmonic expansion to explain shallow inclinations for even the Miocene. In this paper, we develop a simplified statistical model for paleosecular variation (PSV) of the geomagnetic field that can be used to predict paleomagnetic observables. The model predicts that virtual geomagnetic pole (VGP) distributions are circularly symmetric, implying that the associated directions are not, particularly at lower latitudes. Elongation of directions is North-South and varies smoothly as a function of latitude (and inclination). We use the model to characterize distributions expected from PSV to distinguish between directional anomalies resulting from sedimentary inclination error and from non-zero non-dipole terms, in particular a persistent axial octupole term. We develop methodologies to correct the shallow bias resulting from sedimentary inclination error. Application to a study of Oligo-Miocene redbeds in central Asia confirms that the reported discrepancies from a GAD field in this region are most probably due to sedimentary inclination error rather than a non$\mathrm{GAD}$ field geometry or undetected crustal shortening. Although non-GAD fields can be imagined that explain the data equally well, the principle of least astonishment requires us to consider plausible mechanisms such as sedimentary inclination error as the cause of persistent shallow bias before resorting to the very "expensive" option of throwing out the GAD hypothesis.

Timescales of the Paleomagnetic Field

Geophysical Monograph Series 145

Copyright 2004 by the American Geophysical Union 10.1029/145GM08

\section{INTRODUCTION}

One of the most useful assumptions in paleomagnetism is that the geomagnetic field is on average close to that of a geo- 
centric axial dipole (GAD). The GAD model is a specific, testable hypothesis, which for the past few million years provides an excellent fit to global data such that the largest nonGAD contribution is generally found to be no more than about 5\% of GAD (e.g. Opdyke and Henry [1969]; Merrill and McElhinny [1977]; Schneider and Kent [1988]; Gubbins and Kelly [1993]; Kelly and Gubbins [1997]; Quidelleur et al. [1994]; Johnson and Constable [1995]; Johnson and Constable [1998]; Carlut et al. [2000]).

It is difficult to test the GAD hypothesis in ancient times owing to plate movements, rock deformation and remagnetization. Nevertheless, the inescapable conclusion from a variety of paleomagnetic data and analysis techniques is that there is often a strong bias toward shallow inclinations that appears inconsistent with a GAD model for the ancient time-averaged geomagnetic field (e.g., Kent and Smethurst [1998], Westphal [1993], Si and Van der Voo [2001]). There are many potential causes for mean directions and distributions that are biased shallow. Given the fundamental utility of the GAD assumption in paleomagnetism, alternative mechanisms deserve a closer look. In this paper we will consider depositional inclination error, especially in detrital hematite, as a better explanation for many of the discrepant observations.

In this paper, we will examine the evidence for inclination anomalies in the Central Asian redbed sediments and explore the possible explanations. Then we will develop a simple statistical model for paleosecular variation which predicts distributions of geomagnetic vectors in agreement with the currently available data sets. The model can be modified to include arbitrary non-zero gauss terms and we investigate the effect of adding an arbitrary amount of non-zero axial octupole on the predicted distributions. We then consider the consequences of sedimentary inclination error on distributions of directions and propose two methods for detecting and correcting for the resulting shallow bias.

\section{INCLINATION ANOMALIES IN CENTRAL ASIAN RED BEDS}

Paleomagnetic poles obtained from globally distributed locations will tend to average out non-dipole field contributions; hence globally averaged paleopoles should reflect mainly the GAD field. These poles are often used to predict directions at specific locations. The difference between the predicted and observed directions could be caused by local non-dipole field effects, local artifacts caused by rock deformation, or by magnetic recording biases. Paleomagnetists typically assume that the geomagnetic field has been essentially GAD in order to estimate a reference pole for a given plate at the desired time (e.g., Irving [1964]). If the rotation parameters linking various plates are known, then these reference poles can be used to predict directions expected from a GAD field at any place on any linked plate. An updated compilation of paleopoles for the Atlantic-bordering continents for 0-200 Ma shows very good agreement with the GAD model, with only a small $(\sim 3 \%)$ axial quadrupolar contribution [Besse and Courtillot, 2002]. Despite general agreement, comparison of predicted directions with those observed reveals a persistent shallow bias in Cenozoic paleomagnetic directions from sediments of Central Asia (see, e.g., Thomas et al. [1993]; Chauvin et al. [1996]; Cogné et al. [1999]; Si and Van der Voo [2001]; Dupont-Nivet et al. [2002]; Gilder et al. [2003]).

Non-GAD geomagnetic fields, in particular axial octupolar fields $\left(\bar{g}_{3}^{0}\right)$, have been called on to explain the Central Asian inclination anomalies (e.g., Thomas et al. [1993]; Chauvin et al. [1996]; Van der Voo and Torsvik [2001]; Si and Van der Voo [2001]; Dupont-Nivet et al. [2002] ). The logic according to, for example, Si and Van der Voo [2001], is that the reference poles are largely based on results from the UK and North America. A non-zero axial octupolar contribution with the same sign as the dipole makes directions in mid-northern latitudes shallower than expected from a GAD field. These directions, when converted to paleomagnetic poles will be "far-sided." If this reference pole is then used to predict directions in Asia, the predicted directions will be too steep. The effect is amplified by the fact that the actual directions observed in Asia in the same octupolar field will be shallower than expected from GAD. Typical contributions of $\bar{g}_{3}^{0}$ called for are between 10 and $20 \%$ of the average axial dipole.

While most studies attribute the observed inclination shallowing to non-GAD geomagnetic fields, there are alternative interpretations. Cogné et al. [1999] attributed the effect to a large degree of tectonic shortening. Recently, sedimentary inclination error (either by compaction or by initial depositional processes) has gained favor as a possible explanation for the effect (e.g., Gilder et al. [2001]; Dupont-Nivet et al. [2002]; Tan et al. [2003]; Gilder et al. [2003]).

The overwhelming majority of the paleomagnetic data from Central Asia come from red beds whose remanence is attributed to detrital hematite by the authors. Tauxe and Kent [1984] studied natural (modern) and laboratory redeposited sediments with detrital hematite. Results from their redeposition experiments are shown in Figure 1a. These data demonstrate a pronounced bias toward shallow inclinations that follow the "inclination error formula" of King [1955]:

$$
\tan \left(I_{o}\right)=f \tan \left(I_{f}\right)
$$

where $I_{o}$ and $I_{f}$ are the observed and applied field inclinations, respectively, and $f$ is an empirical coefficient (here called the "flattening factor"), estimated to be about 0.55 (dashed line in Figure 1a) in these particular sediments. 

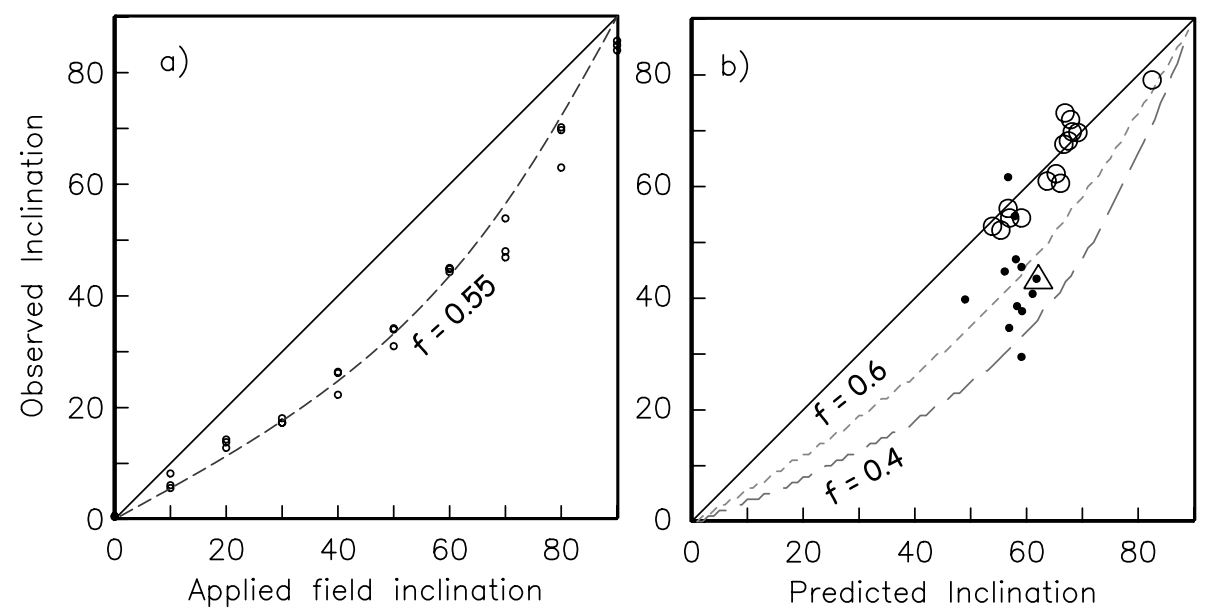

Figure 1. a) Observed inclination versus applied field determined for natural sediments with detrital hematite (data of Tauxe and Kent [1984]). Dashed line is for $f=0.55$. b) Filled (sediments) and open (basalts) circles are observed inclination versus predicted inclination from the APWP for Europe of Besse and Courtillot [2002] for data compiled by Gilder et al. [2003]. Triangle is the magnetostratigraphic study of Gilder et al. [2001] to be discussed later. Dashed lines are the function $\tan \left(I_{o}\right)=f \tan \left(I_{f}\right)$ for $f=0.4$ and $f=0.6$ (as labelled).

Gilder et al. [2003] compiled paleomagnetic data for Cenozoic and Mesozoic sediments and basalts from Central Asia north of the Tibetan plateau. We replot their red bed data compilation as observed inclinations versus predicted inclinations from Besse and Courtillot [2002] as solid dots in Figure 1. Data from igneous rocks are shown as open circles. Theoretical curves for inclination error with $f=0.4$ and 0.6 are shown on Figure $1 \mathrm{~b}$ as dashed lines. In general, the observed inclinations from the sediments fall well below the expected values. Data from basaltic units in the same compilation do not display a shallow bias as shown also by Bazhenov and Mikolaichuk [2002]. These data show conclusively that the sedimentary units are shallower than the basaltic data; hence Gilder et al. [2003] strongly argue for inclination error as a cause of the inclination anomaly.

Based on the predicted and observed inclinations shown in Figure $1 b$, it is reasonable to interpret the shallow inclinations from Central Asian sediments as resulting from sedimentary inclination error. However, non-GAD field geometry or crustal motion inconsistent with geological observations have been called upon as plausible explanations for shallow inclinations in many tectonic studies. Some means for discriminating among the various possibilities would be of general use.

We suggest in this paper that when inclination error occurs, it distorts the original distribution of directions in ways that should be distinguishable from the other mechanisms of inclination shallowing if the characterisics of the data set as a whole are considered. Before we begin to explore the effect of inclination error on distributions of directions, we need to understand what distributions we might expect from secular variation of the geomagnetic field itself. We therefore will first consider statistical paleosecular variation models capable of predicting directions as a function of position on the surface of the Earth. We then will investigate the effect of non-zero average octupolar components and finally we will characterize the effect of sedimentary inclination error on directions.

\section{PALEOMAGNETIC CONSTRAINTS AND STATISTICAL MODELS OF THE GEOMAGNETIC FIELD}

\subsection{The Giant Gaussian Process}

To predict the distribution of directions produced by paleosecular variation of the geomagnetic field, we require a statistical model to generate plausible sets of geomagnetic field vectors. A good starting point is the model of Constable and Parker [1988] (hereafter CP88). This models the time varying geomagnetic field as a "Giant Gaussian Process" (GGP) whereby the gauss coefficients $g_{l}^{m}, h_{l}^{m}$ (except for the axial dipolar term, $g_{1}{ }^{0}$ and in some models also the axial quadrupole term $g_{2}{ }^{0}$ ) have zero mean and standard deviations that are a function of degree $l$. For $l \geq 2$ these standard deviations are given by

$$
\sigma_{l}^{2}=\frac{(c / a)^{2} l \alpha^{2}}{(l+1)(2 l+1)}
$$

where $c / a$ is the ratio of the core radius to that of Earth and $\alpha$ is a fitted parameter. The parameters used in the model of CP88 are listed in Table 1. 
Table 1. Parameters for various PSV models

\begin{tabular}{lcccc}
\hline Parameter & CP88 & CJ98 & QC96 & TK03.GAD \\
\hline $\bar{g}_{1}^{0}$ & $-30 \mu \mathrm{T}$ & $-30 \mu \mathrm{T}$ & $-30 \mu \mathrm{T}$ & $-18 \mu \mathrm{T}$ \\
$\bar{g}_{2}^{0}$ & $-1.8 \mu \mathrm{T}$ & $-1.5 \mu \mathrm{T}$ & $-1.2 \mu \mathrm{T}$ & 0 \\
$\alpha$ & $27.7 \mu \mathrm{T}$ & $15 \mu \mathrm{T}$ & $27.7 \mu \mathrm{T}$ & $7.5 \mu \mathrm{T}$ \\
$\beta$ & & & 3.8 \\
$\sigma_{1}^{0}$ & $0.5 \sigma_{l}=3 \mu \mathrm{T}$ & $3.5 \sigma_{l}=11.72 \mu \mathrm{T}$ & $3 \mu \mathrm{T}$ & $\beta \sigma_{l}=6.4 \mu \mathrm{T}$ \\
$\sigma_{1}^{1}$ & $0.5 \sigma_{l}=3 \mu \mathrm{T}$ & $0.5 \sigma_{l}=1.67 \mu \mathrm{T}$ & $3.0 \mu \mathrm{T}$ & $\sigma_{l}=1.7 \mu \mathrm{T}$ \\
$\sigma_{2}^{0}, \sigma_{2}^{2}$ & $\sigma_{l}=2.14 \mu \mathrm{T}$ & $\sigma_{l}=1.16 \mu \mathrm{T}$ & $1.3 \mu \mathrm{T}$ & $\sigma_{l}=0.6 \mu \mathrm{T}$ \\
$\sigma_{2}^{1}$ & $\sigma_{l}=2.14 \mu \mathrm{T}$ & $3.5 \sigma_{l}=4.06 \mu \mathrm{T}$ & $4.3 \mu \mathrm{T}$ & $\beta \sigma_{l}=2.2 \mu \mathrm{T}$ \\
$l-m$ odd & $\sigma_{l}$ & $\sigma_{l}$ & $\sigma_{l}$ & $\beta \sigma_{l}$ \\
$l-m$ even & $\sigma_{l}$ & $\sigma_{l}$ & $\sigma_{l}$ & $\sigma_{l}$ \\
\hline$c / a=0.547$ & $\left(\sigma_{l}\right)^{2}=$ & $(c / a)^{2 l} \alpha^{2} /[(l+1)(2 l+1)]$ & & \\
\hline \multicolumn{5}{r}{}
\end{tabular}

Many data sets show a persistent offset in equatorial inclinations at least in reverse polarity data sets, consistent with a small non-zero mean axial quadrupolar term $\left(\bar{g}_{2}^{0}\right)$. We are ignoring this effect in the present paper because the bias introduced by $\bar{g}_{2}^{0}$ is negligible for the latitude of the Asian studies and has not been considered as a possible explanation for the inclination anomalies observed there. Hence the version of CP88 and other models discussed here are the "GAD" versions in which the axial quadrupolar term has zero mean (e.g., CP88.GAD).

The advantage of using a statistical model like CP88 is that distributions of directions with various non-zero gauss coefficients (such as the axial quadrupole or octupole term) can be generated and compared with the paleomagnetic observations and with other model predictions. One simply draws coefficients for a field model from gaussian distributions with the specified means and standard deviations and calculates the geomagnetic elements at a given position using the usual formulae (see Constable and Parker [1988] for details). The main disadvantage of the CP88 model is that it fails to account for the observed variations in dispersion of the virtual geo-

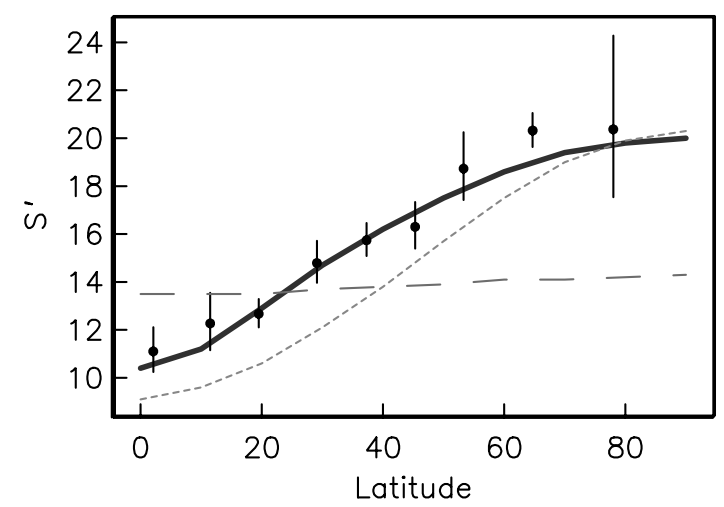

Figure 2. Estimated behavior of $S^{\prime}$ from the data compilation of MM97 (circles). The dashed line is the predicted behavior from CP88.GAD, the dotted line is from CJ98.GAD and the heavy solid line is from TK03.GAD. magnetic poles (VGPs) calculated from directions as a function of latitude (see e.g., McFadden et al. [1988]; Kono and Tanaka [1995]; Constable and Johnson [1999]).

\subsection{VGP Scatter as a Function of Latitude}

McElhinny and McFadden [1997] (hereafter MM97) compiled an updated paleosecular variation of recent lavas (PSVRL) database of directions from lava flows from the last 5 million years that met their minimum acceptance criteria. They also estimated angular standard deviation of the scatter $S$ of the VGPs with latitude. $S$ (e.g., Cox [1969]) is defined as

$$
S^{2}=(N-1)^{-1} \sum_{i=1}^{N}\left(\Delta_{i}\right)^{2}
$$

where $N$ is the number of observations and $\Delta$ is the angle between the $i^{\text {th }}$ VGP and the spin axis. In Figure 2 we show the variation of VGP scatter as a function of latitude from the compilation of MM97 as dots. One criterion for the PSVRL database is that VGPs are rejected if they are more than $45^{\circ}$ from the spin axis in order to avoid over-representation of transitional data. The MM97 estimates of $S$ also used a variable VGP colatitude cutoff as suggested by Vandamme [1994], which is an iterative process whereby the cutoff is found by $\theta=1.85 S+5^{\circ}$. Cutoffs range from $25^{\circ}$ at the equator to $\sim 42^{\circ}$ near the pole. Values of $S$ based on trimmed data sets are here termed $S^{\prime}$. The predicted behavior of $S^{\prime}$ from the CP88.GAD model is shown as a dashed line in Figure 2.

The fact that VGP scatter increases with latitude has been known for decades (e.g., Cox [1962]). As pointed out by McFadden et al. [1988] among others, gauss coefficients that are asymmetric about the equator (those with $l-m$ odd) contribute more strongly to the scatter in VGPs at high latitude than those that are symmetric about the equator (those with $l-m$ even). In order to improve the fit of the statistical paleosecular variation model to their compilation of paleomagnetic observations, Quidelleur and Courtillot [1996] proposed 
Table 2. Predicted observables from the TK03.GAD field model from 10,000 realizations

\begin{tabular}{|c|c|c|c|c|c|c|c|}
\hline$\lambda$ & $\bar{B} \mu \mathrm{T}$ & $\bar{I}$ & $<I>$ & $\kappa$ & $E$ & $S_{l}^{u}$ & $S_{l}^{\prime u}$ \\
\hline 0 & $18.6 \pm 7.4$ & -0.1 & 0 & 15.0 & 2.9 & 14.815 .3 & 10.410 .6 \\
\hline 10 & $19.5 \pm 7.7$ & 17.5 & 19.4 & 15.1 & 2.6 & $\begin{array}{l}14.015 .4 \\
15.616 .0\end{array}$ & $\begin{array}{l}11.211 .4 \\
11.0\end{array}$ \\
\hline 20 & $21.6 \pm 8.6$ & 33.3 & 36.1 & 15.5 & 2.1 & $\begin{array}{l}1 J .016 .0 \\
16.916 .3\end{array}$ & $\begin{array}{l}13.0 \\
12.912 .7\end{array}$ \\
\hline 30 & $24.5 \pm 9.7$ & 46.4 & 49.1 & 16.9 & 1.8 & 18.317 .9 & $14.7_{14.5}^{14.8}$ \\
\hline 40 & $27.5 \pm 10.7$ & 56.9 & 59.2 & 19.4 & 1.5 & 19.619 .9 & 16.216 .4 \\
\hline 50 & $30.6 \pm 11.6$ & 65.4 & 67.2 & 22.6 & 1.3 & 20.720 .4 & $\begin{array}{l}17.517 .4 \\
0\end{array}$ \\
\hline 60 & $33.2 \pm 12.2$ & 72.6 & 73.9 & 26.1 & 1.2 & 21.722 .1 & $\begin{array}{l}18.8 \\
18.618 .4\end{array}$ \\
\hline 70 & $35.2 \pm 12.7$ & 78.9 & 79.7 & 29.1 & 1.1 & 22.422 .8 & $\begin{array}{l}19.6 \\
19.419 .2\end{array}$ \\
\hline 80 & $36.4 \pm 13.0$ & 84.6 & 84.9 & 31.2 & 1.0 & $22.9_{22.5}^{23.2}$ & $19.8_{19.6}^{20.0}$ \\
\hline 90 & $36.8 \pm 13.1$ & 89.8 & 90.0 & 31.8 & 1.0 & 23.0223 .4 & $20.0 \begin{array}{l}20.2 \\
19.8\end{array}$ \\
\hline
\end{tabular}

$\lambda$ : Latitude $\left({ }^{\circ}\right), \bar{B}$ : average field intensity, $\bar{I}$ : average field inclination, $\langle I\rangle$ inclination expected from dipole formula; $\kappa$ : Fisher [1953] precision parameter, $E$ : elongation, $S$ : VGP scatter, untrimmed, $S^{\prime}:$ VGP scatter, trimmed using Vandamme [1994] criterion. A best-fit polynomial to the inclination-elongation data is $E=2.88-0.0087 I-.0005 I^{2}$.

a variation on the CP88 model (hereafter QC96; see Table 1). QC96 improves the fit by decreasing the variance in the $\sigma_{2}^{0}$ (symmetric) term and increasing the variance in the $\sigma_{2}^{1}$ (asymmetric) terms relative to the CP88 model.

The most recent of the GGP type models is that of Constable and Johnson [1999] (hereafter CJ98). CJ98 attempts to fit a compilation of lava flow data for the last 5 million years [Johnson and Constable, 1996]. The variant of their model with zero average for the non-dipole terms is here called CJ98.GAD. Like QC96, CJ98 achieves an increase in high latitude VGP scatter by adding power to the asymmetric terms and decreasing power in the symmetric terms relative to CP88. The prediction of the behavior of $S^{\prime}$ with latitude of CJ98.GAD (CJ98 as in Table 1 but with $\bar{g}_{2}^{0}=0$ ) is shown as a dotted line in Figure 2. It does a good job of predicting the VGP scatter observed at high latitude, but under predicts scatter at lower latitudes. [We note that CJ98 was designed to fit a different data compilation with more stringent VGP co-latitude cut-offs than MM97.]

\subsection{A Simplified Giant Gausssian Process Paleosecular Variation Model}

As discussed in the previous section and seen in Table 1, both QC96 and CJ98 manipulate the variance for each gauss term separately to achieve a fit to the paleomagnetic observations. We propose here a return to the simplicity of CP88, but modify it to properly account for the observed dependence of $S$ on latitude. The latitudinal dependence of $S$ can be simulated by having larger variance in the asymmetric gauss coefficients than in the symmetric ones. We therefore fit the first order properties of the paleosecular variation data base (average intensity and dispersion of VGPs with lati- tude) with three parameters: the average axial dipole term $\bar{g}_{1}^{0}, \alpha$ as in Equation 2 and $\beta$ defined as the ratio $\frac{\sigma_{l}^{m}(l-m \text { :odd })}{\sigma_{l}^{m}(l-m: \text { even })}$ for a given degree $l$.

Because our understanding of the average field intensity has changed recently (e.g., Selkin and Tauxe [2000]), none of the statistical models fit the observed average intensity of the magnetic field very well, having an average field approximately equal to the present field. Selkin and Tauxe [2000] arrived at an average of approximately half that value, that is, the virtual axial dipole moment (VADM) of the present field is approximately $80 \mathrm{ZAm}^{2}\left[\mathrm{NB}: \mathrm{Z}=10^{21}\right.$ ] whereas the average VADM for the last five million years is approximately $46 \mathrm{ZAm}$. We therefore set the value of the average axial dipole term $\left(\bar{g}_{1}^{0}\right)$ to give the correct average VADM (see Table 1).

Once the value for the axial dipole term has been set, $\alpha$ has a strong effect on the scatter of VGPs observed at equatorial latitudes. Having changed $\bar{g}_{1}^{0}$, then, we must also change $\alpha$ if we are to fit the data at least as well as prior models. As noted previously, $\beta$ has the strongest effect at high latitudes. Therefore we chose $\alpha$ and $\beta$ to give the best fit to the VGP scatter as estimated by MM97. Our preferred values are listed in Table 1 and the fit of predicted $S^{\prime}$ from 10,000 realizations to the dataset of MM97 is shown as the heavy solid line in Figure 2. (Both trimmed $\left(S^{\prime}\right)$ and untrimmed $(S)$ estimates are listed in Table 2; trimming typically reduces $S$ by about $2^{\circ}$.) As it was designed to do, the TK03.GAD model fits the data of MM97 very well.

A departure from previous models in the TK03.GAD model is that the axial dipole term $\left(g_{1}^{0}\right.$; asymmetric degree one term) is no longer treated specially apart from its non-zero mean value; its variation is treated identically to other terms. We plot the values for $\sigma_{l}^{m}$ for the two families (symmetric and asymmetric) in Figure $3 \mathrm{a}$. 

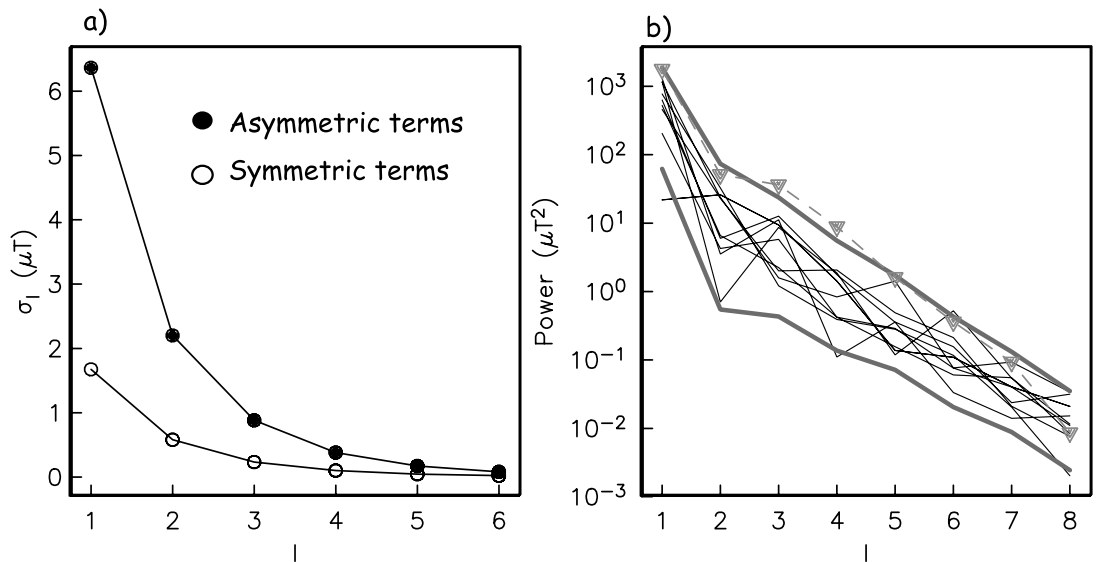

Figure 3. a) Variation of $\sigma$ as a function of degree $l$ for the symmetric $(l-m$ even $)$ and asymmetric $(l-m$ odd $)$ gauss terms for model TK03.GAD (see Table 1). b) Power evaluated for representative realizations of the TK03.GAD (thin lines). 95\% confidence bounds derived from 10,000 realizations (heavy lines). Power spectrum of the IGRF for 1995 (dashed line with triangles).

\subsection{Lowes Spectrum}

Constable and Parker [1988] used the present (1980 International Geomagnetic Reference Field) as a guide for constructing CP88. TK03 is constructed to fit the paleomagnetic data for the last 5 million years instead. To compare the statistical behavior of model TK03.GAD with the present geomagnetic field, we can calculate the power $R_{i}$ in each degree (see, e.g., CP88) using the formula of Lowes [1974]:

$$
R_{i}=\sum_{m=0}^{l}(l+1)\left[\left(g_{l}^{m}\right)^{2}+\left(h_{l}^{m}\right)^{2}\right]
$$

for realizations of the model. We plot 25 so-called "Lowesspectra" as thin dashed lines in Figure 3b. Averaging 10,000 such realizations gives $95 \%$ confidence bounds on the model which are plotted as heavy lines in Figure 3b. We also plot the Lowes spectrum of the 1995 International Geomagnetic Reference Field as triangles. The fact that the spectrum of the present field lies at the very upper bound of realizations from our statistical field model supports the contention of Hulot and Gallet [1996] that the present field is not a good guide for the time-averaged field. In fact, it appears to be quite an unusual field state.

\subsection{Predicted Distributions of Geomagnetic Vectors}

Geomagnetic field vectors evaluated at various latitudes $(\lambda)$ from 1000 realizations of model TK03.GAD are shown in Plate 1a-d. We have plotted each realization as a vector end-point in three dimensions where the RGB color value reflects the contributions of North (red), East (green) and Down (blue). The same realizations are plotted along the principal directions of each cloud of points in Plate $1 \mathrm{e}-\mathrm{h}$. This projection is in many ways similar to an equal area projection of unit vectors centered on the principal direction, but we have included the intensity information as well.

The fact that we can simulate the full geomagnetic field vector allows us to predict the behavior of various parameters in frequent use in paleomagnetic studies, for example VGPs and virtual dipole moments (VDMs). We convert intensity values from 1000 realizations of TK03.GAD to Virtual Axial Dipole Moments (VADM) and plot them against the VGP latitude of the associated direction (Figure 4). This plot exhibits the well known pattern from the geomagnetic field (e.g., Tanaka et al. [1995]) of low VADMs associated with low VGP latitudes. We note that while low paleointensities fre-

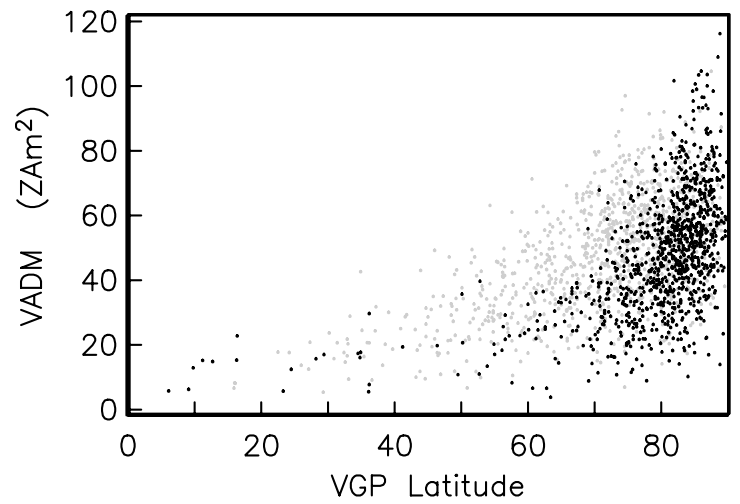

Figure 4. Vectors from realizations of TK03.GAD converted to VGP latitude and VADM for the equator (black) and the polar (lighter) observation sites. 

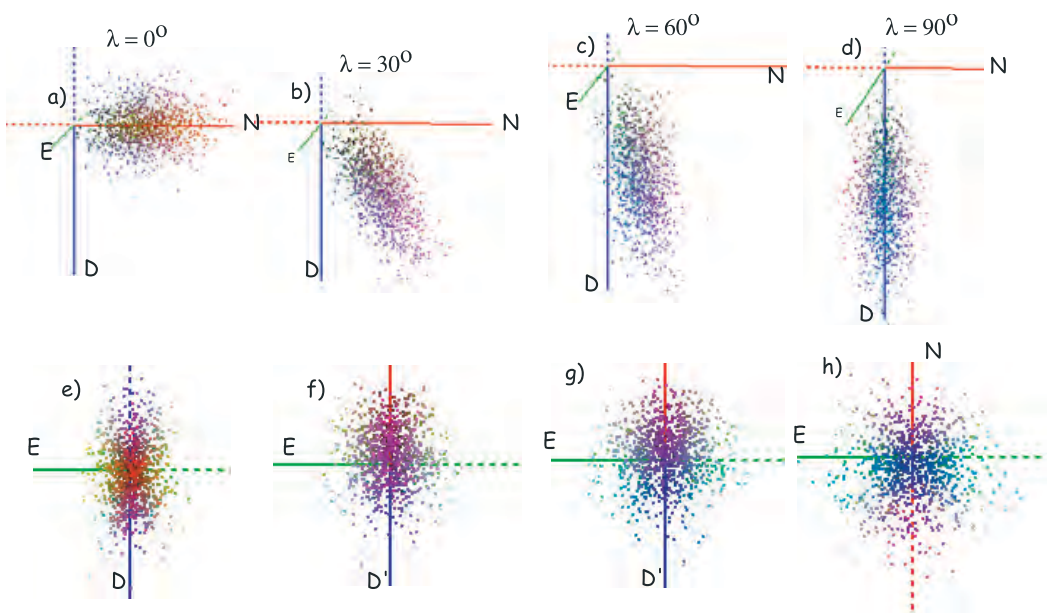

Plate 1. 1000 realizations of TK03.GAD projected as North (red), East (green) and Down (blue) components. Each dot is assigned the RGB color corresponding to the contributions from each component. a-d) All North axes are $40 \mu \mathrm{T}$ long. (South, East and Up axis are the dashed lines. a) Equator, b) $30^{\circ} \mathrm{N}$, c) $60^{\circ} \mathrm{N}$, d) $90^{\circ} \mathrm{N}$. e-h) Same data as a-d) but projected along the principal axis for each data cloud. All East axes are $20 \mu \mathrm{T}$. Axes labelled $D^{\prime}$ are projections in the N-S plane looking along the expected direction at that latitude. e) Equator, f) $30^{\circ} \mathrm{N}$, g) $60^{\circ} \mathrm{N}$, h) $90^{\circ} \mathrm{N}$.
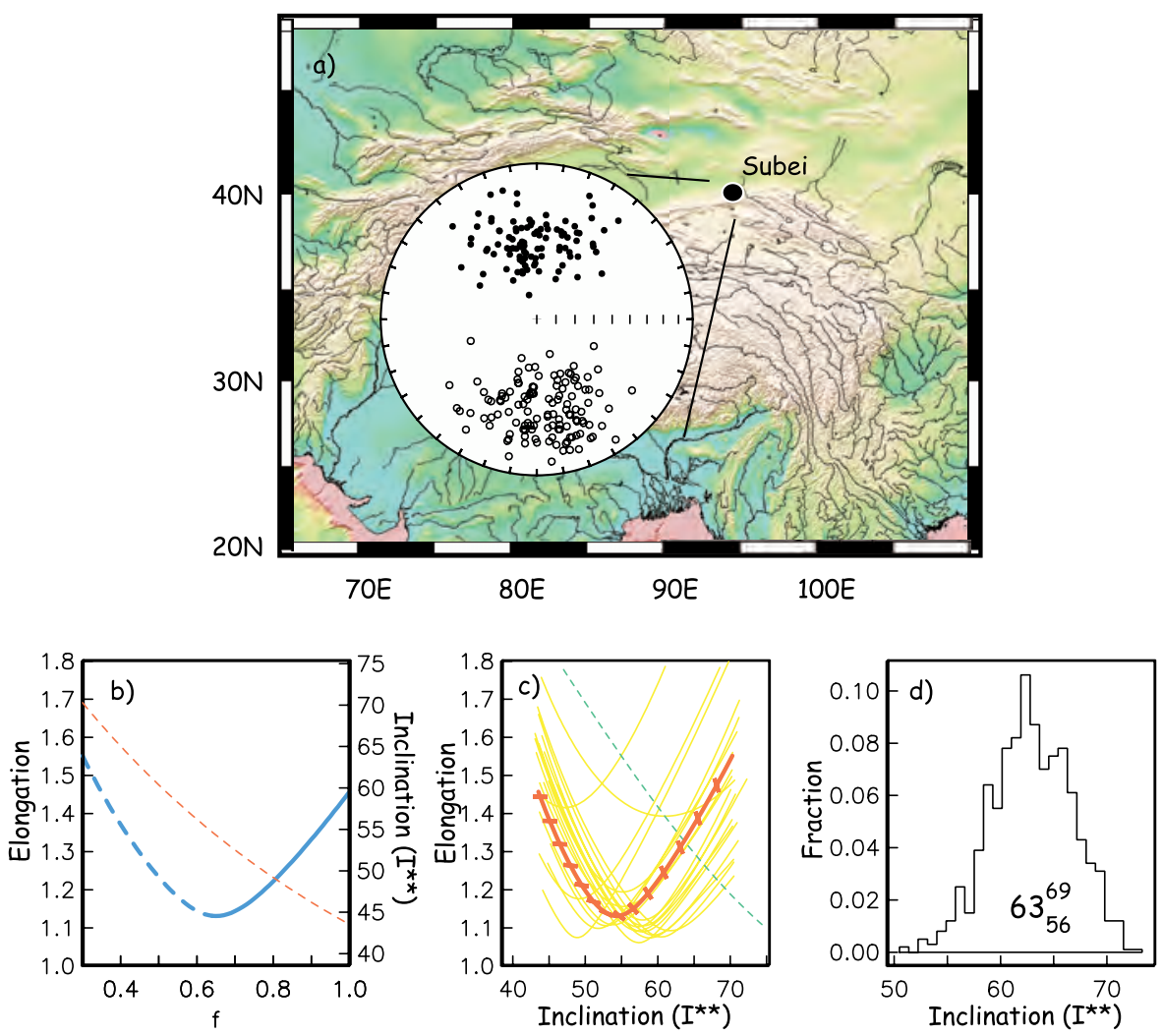

Plate 2. a) Paleomagnetic directions of Oligo-Miocene redbeds from Asia [Gilder et al. 2001] in equal area projection (stratigraphic coordinates). b) Plot of elongation (heavy solid and dashed line) and inclination (dashed) as a function of unflattening by the parameter $f$ in Equation 2. Elongation is $\mathrm{E}-\mathrm{W}(\mathrm{N}-\mathrm{S})$ when heavy line is solid (dashed) c) Plot of elongation versus inclination for the data in b) (solid) and for the TK03.GAD model (dashed). Also shown are results from 20 bootstrapped datasets. The crossing points represents the inclination/elongation pair most consistent with the TK03.GAD model. d) Histogram of crossing points from 1000 bootstrapped datasets. The most frequent inclination $\left(63^{\circ}\right)$ is exactly that predicted from the Besse and Courtillot [2001] European APWP. The 95\% confidence bounds on this estimate are 56-69. 
quently occur with no directional deviations, all highly divergent directions are associated with low paleointensity. It is therefore perhaps inadvisable to identify "excursions" on the basis of intensity records alone as excursions are by definition intervals of deviant observation sites directions. The lighter points in Figure 4 are from observations sites at the pole, while the darker (black) points are evaluated at the equator. There are many more divergent VGP latitudes in the polar simulations than at the equator from the same field models. This model would predict therefore that excursions would only rarely be observed globally, as deviant directions (defined as $>45^{\circ}$ from the pole) are much more prevalent at high latitude observation sites than at low latitude observation sites in the model. Furthermore, our model suggests that the initial selection procedure of MM97 would exclude many observations from high latitude sites while including the comparable observations from the same field state observed at low latitudes.

Because of the comparative dearth of intensity information in routine paleomagnetic data sets, paleomagnetists rarely consider both direction and intensity in a single plot. Plots similar to those shown in Plate 1 cannot be constructed from the current data base with enough data points to fully characterize the vector distribution of the paleomagnetic field as
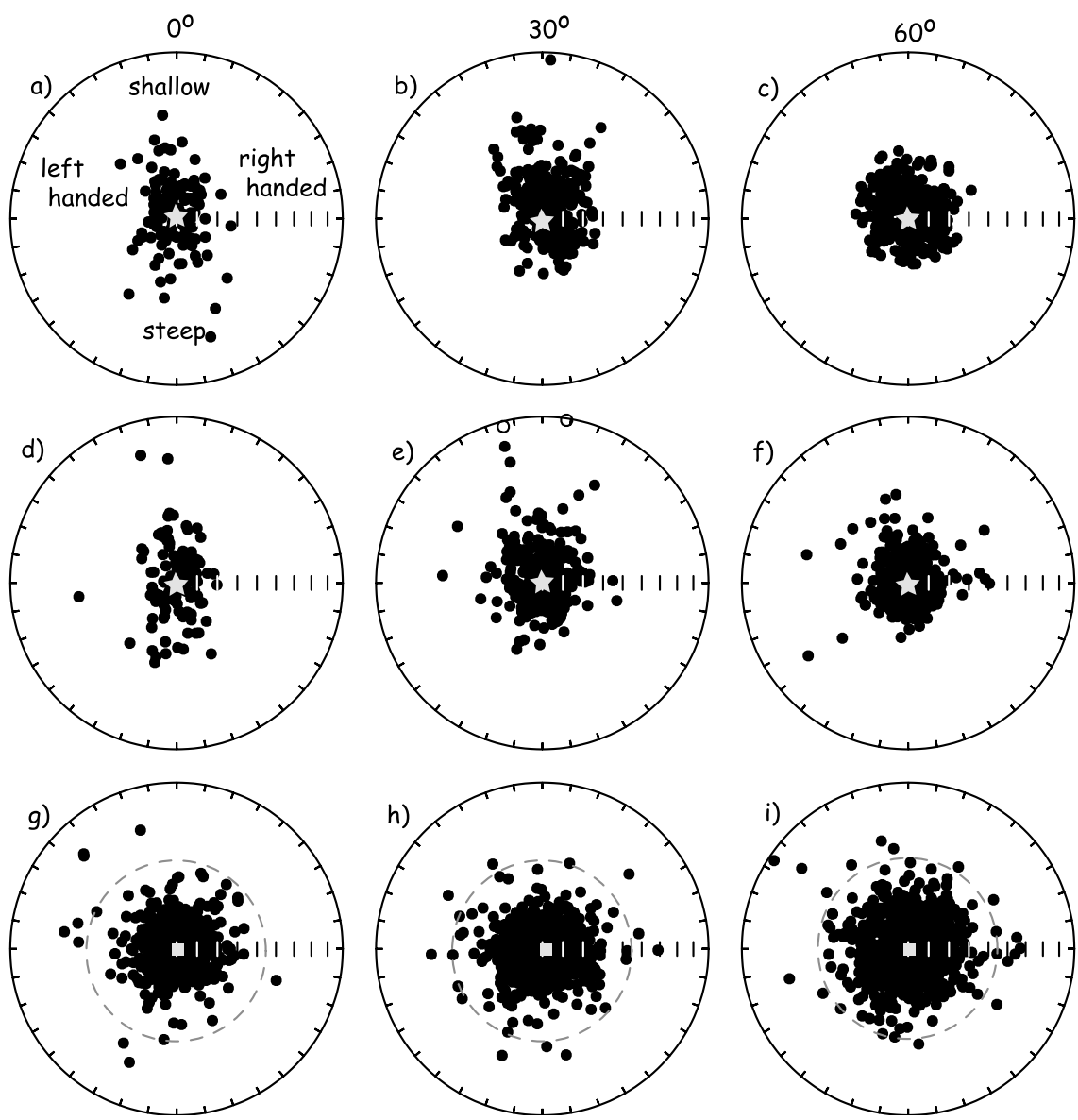

Figure 5. a) Paleomagnetic directions from the PSVRL database (see McElhinny and McFadden [1997]) compiled for latitude band $0-5^{\circ}(\mathrm{N} \& \mathrm{~S})$. Antipodes of reverse directions are used. The expected direction is at the star at the center of the equal area projection. Directions in the upper (lower) half are shallower (steeper) than expected and those to the right (left) are right-handed (left-handed). b) Same as a) but for $25-35^{\circ}$ (N\&S) latitude band. c) Same as a) but for $55-65^{\circ}$ (N\&S) latitude band. d) Same as a) but directions are from realizations of the TK03.GAD model evaluated at $0^{\circ}$ latitude. There are the same number of directions as in a). e) Same as b) but for TK03.GAD model at $30^{\circ}$ latitude. f) Same as c) but for the TK03.GAD model at $60^{\circ}$ latitude. $g-i$ ) The associated VGP positions of the model realizations of $d-f$ ) plotted in polar projection (squares are the poles). The dashed circle is the $45^{\circ}$ cutoff used as an initial cutoff for entry into the PSVRL database. All VGPs outside of this circle would have been eliminated as "transitional" or "excursional". Calculations of $S^{\prime}$ eliminate additional VGPs based on the variable cutoff criterion (see text). 

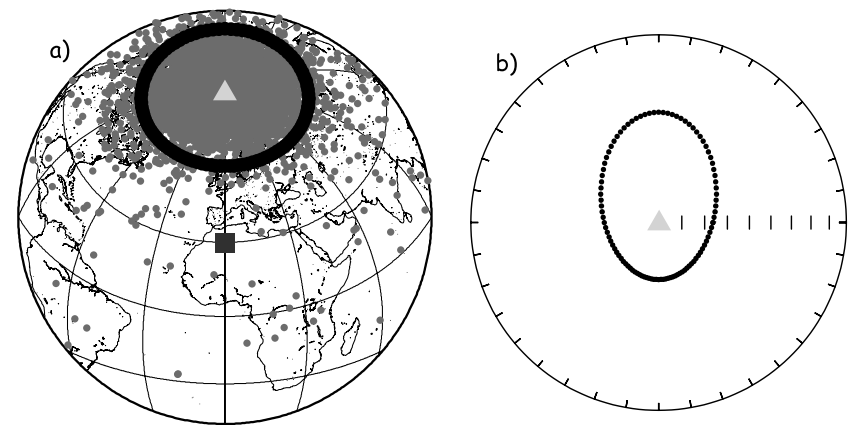

Figure 6. a) VGPs from geomagnetic vectors evaluated at $30^{\circ} \mathrm{N}$ (site of observation shown as square). The geographic pole is shown as a triangle. A set of VGP positions at $60^{\circ} \mathrm{N}$ are shown at the site of observation [squares in (a)] converted from the black ring. b) Directions observed at the site of observation square in a) converted from black ring of VGPs in a) which correspond to the VGP positions at $60^{\circ} \mathrm{N}$. These directions have been projected along expected direction at site of observation (triangle). Note that a circularly symmetric ring about the geographic pole gives an asymmetric distribution of directions with a shallow bias.

a function of latitude. Instead, paleomagnetists generally plot directional data as unit vectors in equal area projection.

To illustrate how directions behave as a function of latitude, we plot directional data selected from the PSVRL database (downloaded in January 2002 from the NGDC website) for $10^{\circ}$ latitude bands in Figures $5 \mathrm{a}-\mathrm{c}$. The directions are plotted (taking the antipodes of the reverse directions) projected in equal area projections along the expected direction at each latitude from a GAD field (Hoffman [1984]). In addition to the criteria of MM97 for inclusion in the database, we selected data with demagnetization codes of 2 or better from sites with at least 3 specimens and a $\kappa$ of at least 100 . We show realizations of the same number of directions drawn from TK03.GAD (Figures $5 \mathrm{~d}-\mathrm{f}$ ) and the associated VGPs (Figures $5 \mathrm{~g}-\mathrm{i}$ ). Note that no VGPs generated from TK03.GAD were trimmed in these plots.

One observation from model TK03.GAD is that the simulated distributions of VGPs are circularly symmetric at all latitudes. [NB: The VGP distributions are not Fisherian sensu strictu as the distribution of latitudes is not exponential, having a low latitude tail.] Circular symmetry of VGPs implies that the corresponding distributions of directions cannot be symmetric everywhere. In fact there is no essentially dipolar field structure that can give rise to Fisher distributed directions everywhere, so it is generally true that data sets of geomagnetic field directions would not be expected to be Fisher distributed. Although this has long been suspected (e.g., Creer [1959]), it has been largely ignored in routine paleomagnetic studies (but see important exceptions by Baag and Helsley [1974], Kono [1997], Beck [1999], and Tanaka [1999]).
An immediate consequence of circularly symmetric VGPs is illustrated in Figure 6a in which we plot as small dots the VGP positions from field vectors drawn from TK03.GAD evaluated at the sampling site $\left(30^{\circ} \mathrm{N}\right.$; square). The geographic pole is indicated by the triangle. We also show a black ring of VGP positions at $60^{\circ} \mathrm{N}$. This ring is converted to the expected direction at the sampling site in Figure $6 \mathrm{~b}$ with the expected direction at the center of the diagram. The ring of VGPs maps into an ellipse that is asymmetrical with a significant shallow bias. Because the most shallow directions are associated with the low intensities (see e.g., Plate $1 \mathrm{~b}$ and Figure 4), they do not bias the vector mean significantly. They do, however, bias the average inclination derived from unit vectors (see Table 2). This inclination anomaly varies from zero at the equator to a maximum of about $3^{\circ}$ at midlatitudes and was predicted by Creer [1983] from a secular variation model based on migrating radial dipoles. The essential feature of Creer's model was that VGP distributions are circularly symmetric which is also a key feature of the types of models considered here. Also noted by Creer [1983], the inclination anomaly has the same form as a non-zero contribution of the $g_{3}^{0}$ term. The magnitude of the effect is not large enough, however, to explain inclination anomalies of $\sim 20^{\circ}$ under consideration here.

To characterize the elongation of the distribution of directions derived from Fisher distributed VGPs as a function of latitude, Tanaka [1999] used the ratio of the 95\% confidence radii $\alpha_{31} / \alpha_{32}$ from Bingham statistics [Bingham, 1964; Bingham, 1974]. The radii of the Bingham ellipses are ultimately based on the eigenvalues of the "orientation matrix" $\mathbf{T}[$ Scheidegger, (1965)] which is defined as:

$$
\mathbf{T}=\left(\begin{array}{lll}
\sum x_{1 j} x_{1 i} & \sum x_{1 j} x_{2 i} & \sum x_{1 j} x_{3 j} \\
\sum x_{1 j} x_{2 j} & \sum x_{2 j} x_{2 j} & \sum x_{2 j} x_{3 j} \\
\sum x_{1 j} x_{3 j} & \sum x_{2 j} x_{3 j} & \sum x_{3 j} x_{3 j}
\end{array}\right) .
$$

where $x_{i j}$ are the $i^{t h}$ component of the $j^{\text {th }}$ unit vector. The eigenvalues $\tau_{i}$ and eigenvectors $\mathbf{V}_{i}$ reflect the shape and orientation of the distribution of directions, respectively. For Fisher distributions, the eigenvector $\mathbf{V}_{1}$ associated with the maximum eigenvalue $\tau_{1}$ is coincident with the Fisher mean direction. $\mathbf{V}_{2}$ and $\mathbf{V}_{3}$ are in the directions of the major and minor axes of the Bingham confidence ellipse whose radii are related through a non-linear transformation to the eigenvalues. Here we use the eigenvalues themselves and follow Tauxe [1998] who defined an elongation parameter $E$ as the ratio $\tau_{2} / \tau_{3}$ to quantify the asymmetry in the distributions of directions seen in both the PSVRL dataset and the TK03.GAD model (Figure $5 \mathrm{a}-\mathrm{f}$ ). (Note that this is different from the elongation defined later by Beck [1999]). The elongation direction is the declination of $\mathbf{V}_{2}$ or $D \mathbf{V}_{3}$. 
We plot the behavior of elongation for the PSVRL data compilation in approximately $20^{\circ}$ latitude bands in Figure 7 as solid dots. The dots are placed at the average latitude of the data set and the horizontal bars indicate the latitude window from which the data were drawn. Also shown is the variation of $E$ predicted from TK03.GAD (triangles in Figure 7). $E$ varies in the TK03.GAD model from $\sim 3$ (rather elongate) at the equator to unity (approximately symmetric) near the poles (see also Table 2 ). $D_{\mathbf{V}_{2}}$ remains essentially zero for all distributions that have significant elongation. In other words, the distributions of field directions tend to be elongated in the North-South direction. The distributions of VGPs, however, remain highly symmetric (see Figure $5 \mathrm{~g}-\mathrm{i}$ ). We also show the variation of elongation with latitude from the CJ98.GAD model (circles) for comparison. Even with quite different statistical behavior of the field, the variation of $E$ with latitude is rather similar. We also plot the inclination variation with latitude $(\lambda)$ predicted from the dipole formula $\tan I=2 \tan \lambda$ as squares in Figure 7.

As an aside, given the expectation for elliptical distributions of directions derived from inherently GAD fields, it is likely to be inappropriate to use Fisher statistics on directional data sets. Love and Constable [2003] offer a means for incorporating intensity information into the averaging process, but as yet have only dealt with the isotropic case. A glance at Plate 1 suggests that distributions of paleomagnetic vectors are unlikely to be isotropic (which would have data clouds that are "round" as opposed to the triaxial distributions observed here) and there is a need for anisotropic statistical methods for dealing with geomagnetic vector data. Until the theory is more developed, a non-parametric bootstrap (see Tauxe [1998]) is probably the least biased way to get confidence intervals for distributions of directions or their components.

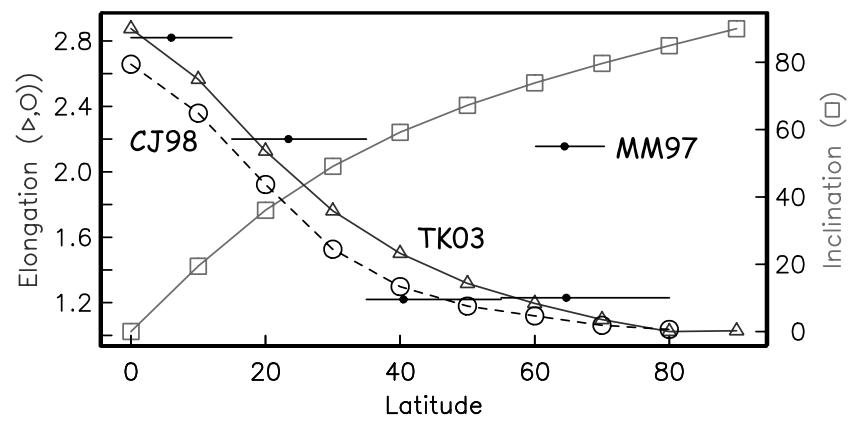

Figure 7. Variation of elongation (triangles) and average inclination (squares) versus latitude for the TK03.GAD model. Also shown is elongation from CJ98.GAD (circles) and the selected directions from the PSVRL database (see text). By about $60^{\circ} \mathrm{N}$ latitude, distributions of directions are virtually circularly symmetric.

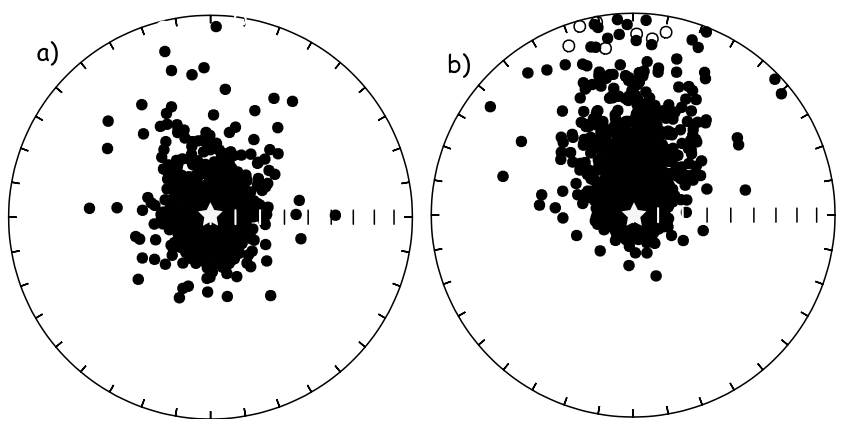

Figure 8. Equal area projections as in Figure 5. Sets of directions evaluated for 30 latitude, projected along direction expected from a GAD field. a) Directions drawn from TK03.GAD. b) Directions drawn from TK03.g30 $\left(\bar{g}_{3}^{0}\right.$ set to $\left.20 \% \bar{g}_{1}^{0}\right)$.

\subsection{Contribution of Non-Zero Mean Octupolar Term}

We are interested in this paper in the difference between directional dispersion that results from non-GAD contributions (in particular the octupole) and dispersion that comes from sedimentary processes. Therefore, it is worth considering what effect the axial octupolar contribution $\left(\bar{g}_{3}^{0}\right.$, frequently called upon to explain the inclination anomalies in the ancient field) would have on directions observed in the paleomagnetic field. In Figure 8 we illustrate the effect of non-zero octupolar components on the distribution of directions observed at $30^{\circ}$ latitude. Figure 8 a shows the distribution of directions drawn from TK03.GAD as viewed down the expected direction from a GAD field. Figure $8 \mathrm{~b}$ shows TK03, but with the $\bar{g}_{3}^{0}$ term set to $20 \%$ of $\bar{g}_{1}^{0}$ (TK03.g30). The average inclination of this set of directions is $30.4^{\circ}$, compared to $49^{\circ}$ expected from the dipole formula (see Table 3 ). In general, the addition of a non-zero axial octupolar component of the same sign as $\bar{g}_{1}^{0}$ at mid latitude tends to increase the elongation in the $\mathrm{N}-\mathrm{S}$ direction and decrease the average inclination. As noted earlier, this has an identical form to the bias that results from neglecting the intensity information. However, the inclination anomaly of Central Asian red beds is $\sim 20^{\circ}$ at $40^{\circ} \mathrm{N}$, far larger than can be achieved by ignoring intensity; one requires a non-zero mean contribution of $10-20 \%$ for the $g_{3}^{0}$ term to explain the observation.

Table 3. Predicted observables from the TK03.g30 field model with nonzero $g_{3}^{0}$ mean evaluated at $30^{\circ} \mathrm{N}$. See caption for Table 2 .

\begin{tabular}{lcc}
\hline$\% g_{3}^{0}$ & $\bar{I}$ & $E$ \\
\hline 5 & 42.6 & 2.0 \\
10 & 38.4 & 2.3 \\
15 & 34.1 & 2.6 \\
20 & 29.6 & 3.0 \\
30 & 20.3 & 3.9 \\
\hline
\end{tabular}




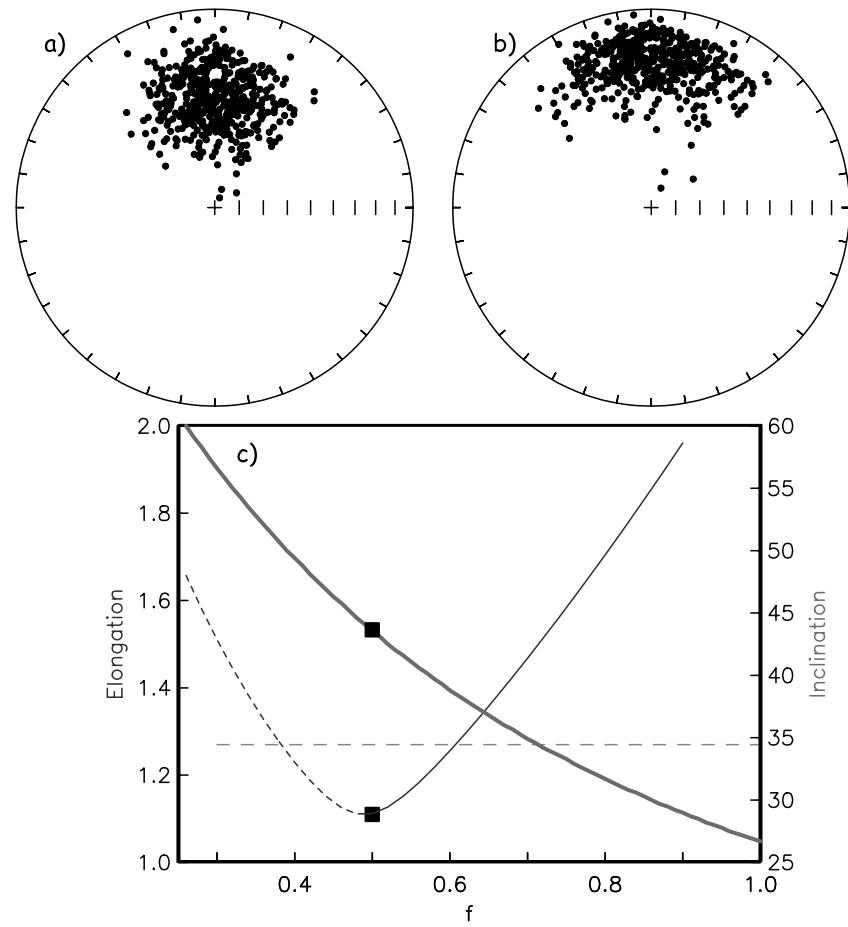

Figure 9. a) Equal area projections of 500 directions drawn from a Fisher distribution with $\kappa=20, \bar{D}=0^{\circ}, \bar{I}=45^{\circ}$. Center of diagram is the vertical. b) Directions from a) with inclination distorted by function $\tan \left(I_{o}\right)=f \tan \left(I_{f}\right)$, setting $f=0.5$. c) Elongation (solid line with dashed extension) and inclination (heavy solid line) as a function of the transformation to "undo" the inclination error (see text). The elongation changes from East-West (solid portion of elongation curve) to North-South (dashed portion) at about $f=0.5 .95 \%$ of data sets drawn from Fisher distributions with $N=500, \kappa=20$ have elongations below the horizontal dashed line $(E=1.27)$. The original elongation (inclination) values, $1.11\left(43^{\circ}\right)$, are plotted as black squares.

\subsection{Sedimentary Inclination Error}

We are now in a position to examine the effect of sedimentary flattening "inclination error" of, e.g., King [1955]) on various distributions of directions. To investigate the effect of inclination error on a set of directions, we draw 500 directions from a Fisher distribution [Fisher, 1953] with a precision parameter $\kappa$ of 20 , a true mean declination $\hat{D}=0$ and an inclination of $\hat{I}=45$. [We use the program fishrot in the PMAG1.7 software distribution available at http://sorcerer.ucsd.edu/software/.] The calculated mean direction of the data set is $\bar{D}=0.1^{\circ}, \bar{I}=43.6^{\circ}, \alpha_{95}=1.4^{\circ}$ (see Figure 9a).

We transformed each inclination $(I)$ of this data set to a new inclination $\left(I^{*}\right)$ by the "inclination error" formula (Equation 1) with $f=0.5$. The transformed directions $(D$, which remains the same) and $I^{*}$ are shown in Figure 9b. The new distribution has a flattened mean inclination of $\bar{I}^{*}=26.7^{\circ}$, and is clearly distorted from a Fisher distribution with a pronounced East-West elongation.

To assess the degree of asymmetry in the directions, we use the eigenanalysis of the orientation tensor as before. In a Fisher distribution, eigenvalues $\tau_{2}$ and $\tau_{3}$ are statistically indistinguishable making the distribution of data symmetric about the principal direction ( $E$ is close to unity). [Monte Carlo simulation of 1000 Fisher distributions with $N=500$, $\kappa=20$ have $\mathrm{E}<1.195 \%$ of the time.] If we suppose that the asymmetry in a given data set was caused by "inclination error" acting on an initially symmetric distribution, we could invert the data by:

$$
\tan \left(I^{* *}\right)=(1 / f) \tan \left(I^{*}\right)
$$

Calculating the eigenparameters for a variety of values of $f$ would allow us to determine the value of $f$ that brings the data to minimum elongation.

Results of such an inversion on the distorted data of Figure $9 b$ are shown in Figure 9c in which we plot the elongation (dashed and solid line) and mean inclination (heavy solid line) as a function of $f$. The value of $f$ that achieves minimum elongation is $f=$ 0.5 . The mean direction of the inverted data set using $f=0.5$ is of course identical to the original in this example.

\section{DETECTION/CORRECTION OF INCLINATION ERROR}

\section{1. "Correction-by-site" Method}

While the distribution of directions derived from the geomagnetic field is unlikely to be Fisher distributed except at high latitude, the individual sample directions from each site are in fact expected to be Fisher distributed. Random perturbations in the recording and orienting processes will predominate over field variations in the short time span represented by the site. Therefore, if one had enough samples per site, one could seek the $f$ that minimizes $E$ at a site level, find $D, I^{* *}$ (using Equation 3) and recalculate the site means based on the $D$, $I^{* *}$ sample directions.

We illustrate the so-called "correction-by-site" (CBS) procedure for a hypothetical study in Figure 10. In Figure 10a, we show the set of 100 directions drawn from TK03.GAD evaluated at $30^{\circ} \mathrm{N}$ (the large dots; drawn from those shown in Figure 5e). The average of these is $\bar{D}=358.7^{\circ}, \bar{I}=46.3^{\circ}$. For each of these "sites", we draw 20 "sample" directions from a Fisher distribution with $\kappa=100$, shown as small dots. We transformed each sample direction using the inclination error formula with a flattening factor $f$ of 0.5 . The transformed $D, I^{*}$ are shown as small dots in Figure 10b. The average of the "flattened" site means (shown as large dots) is $\bar{D}=358.8^{\circ}, \bar{I}^{*}=29.1^{\circ}$. 


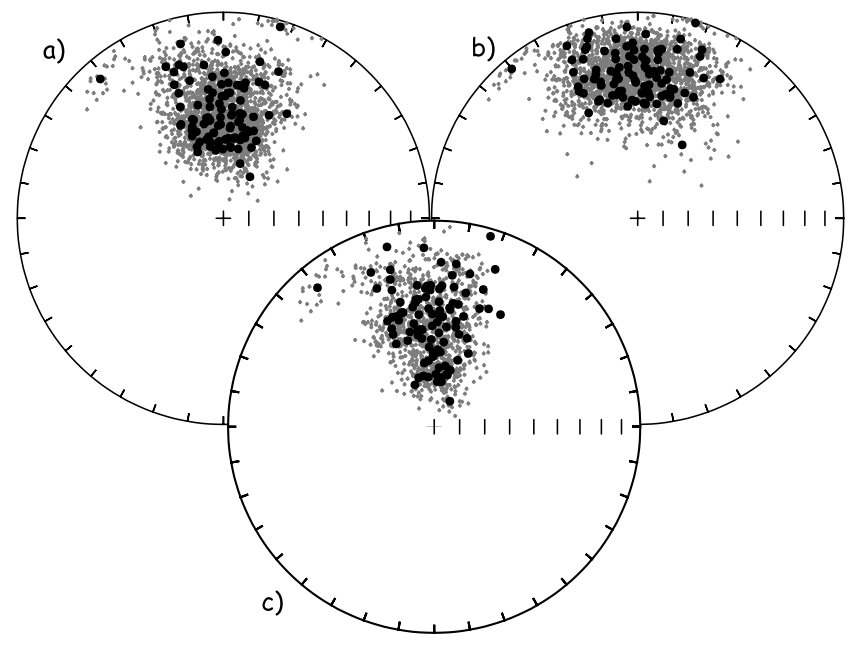

Figure 10. a) Hypothetical Fisher distributed sample directions (small dots) for each site mean (large dots) simulated for 100 hypothetical sites whose directions were drawn from TK03.GAD at $30^{\circ} \mathrm{N}$. There are 20 samples at each site. b) Data from a) after transforming to $I^{* *}$ using $f=0.5$. c) Data from b) after seeking the value of $f$ that minimizes $E$ within a site, inverting for $I^{* *}$ using that $f$ in Equation 3. Each "site" mean was recalculated with the $D, I^{* *}$ for each sample.

The data from each site were treated as in Figure $9 \mathrm{c}$ to find the value of $f(1.0>f>0.3)$ which minimized elongation. After finding the optimum $f$ at each site, we inverted the sample directions using Equation 3. These $D, I^{* *}$ are shown as small dots in Figure 10c. New average values for each site are shown as large dots and the mean of these sites is $\bar{D}=358.7^{\circ}, \bar{I}=46.3^{\circ}$, virtually identical to the original value.

Our CBS method relies on a few essential assumptions. First, we assume that sample directions at a site level are Fisher distributed and that sufficient samples were obtained to adequately represent that distribution. We assume that every sample at a given site was affected by the identical flattening factor. We do not, however, need to assume any $a$ priori distribution of the original geomagnetic field directions. Success of the method will depend on taking enough samples at the site level and sampling uniform enough lithology that the samples single value of $f$ assumption is reasonable. Based on Monte Carlo simulations, we estimate that perhaps as many as 20 are necessary for a robust estimate of $f$. Similar arguments at the study level by Tauxe et al. [2003] suggest that at least 100 sites are necessary to represent the distribution of directions drawn from plausible models of the geomagnetic field.

\section{2. "Elongation/Inclination Method"}

Unfortunately, the generally available databases do not yet retain data at the sample level, nor do most studies have both large numbers of sites $(\geq 100)$ and large numbers of samples per site $(\geq 20)$. However, one can seek the value of $f$ at a study level that yields an elongation/inclination pair consistent with some geomagnetic field model. We illustrate this "elongation/inclination" $(E / I)$ method in the following.

The $E / I$ method of inclination correction requires a data set large enough to have sampled secular variation of the geomagnetic field and one in which an average value of $f$ can reasonably be estimated for the entire study. Most studies aimed at producing paleomagnetic poles are too small, typically having a few dozen sites. Fortunately, the magnetostratigraphic data set of Gilder et al. [2001] is unusually large, having 222 sites. [There are only $\sim 2$ samples per site, however, so we are unable to test the CBS method with this data set.] Directions from this study are shown in Plate 2a. These have a mean of $\bar{D}=356.1^{\circ}, \bar{I}=43.7^{\circ}$. The initial distribution is elongated $\mathrm{E}-\mathrm{W}$, which immediately suggests that the anomalously shallow mean inclination is unlikely to be due to a geomagnetic field with a significant axial octupolar contribution because that always produces $\mathrm{N}-\mathrm{S}$ elongation.

Assuming that the location of the study (presently located at $39.5^{\circ} \mathrm{N}, 94.7^{\circ} \mathrm{E}$ ) has been fixed to the European coordinate system and taking the $20 \mathrm{Myr}$ pole for Europe from Besse and Courtillot [2002] $\left(81.4^{\circ} \mathrm{N}, 149.7^{\circ} \mathrm{E}\right)$, the inclination is predicted to be $63^{\circ}$ (see triangle in Figure 1b). These sediments are typical of Asian sedimentary units in having an inclination relative to the predicted values that is some $20^{\circ}$ too shallow.

To find the average value of $f$ appropriate for the study using the elongation/inclination method we apply Equation 3 to the data shown in Plate 2a (taking the antipodes of the reverse directions) for a range of values of $f$ (Plate $2 b$ ). In Plate $2 \mathrm{c}$, we plot the elongation versus inclination for each set directions transformed using a given value of $f$. These are plotted along with the elongation/inclination behavior predicted by TK03.GAD. The orientation of $D_{\mathbf{V}_{2}}$ is shown as hatchures on curve for the data (heavy line) in Plate $2 \mathrm{c}$, with vertical lines being $\mathrm{N}-\mathrm{S}$ and horizontal lines being $\mathrm{E}-\mathrm{W}$. A best-fit polynomial to the model inclination-elongation data in Table 2 is: $E=2.88-0.0087 I-0.0005 I^{2}$ and is plotted as a dashed line in Plate 2c. The model (dashed line) and observed elongation/inclination (heavy hatched) curves cross at an inclination of $\sim 64^{\circ}$.

To obtain confidence bounds on the "corrected" inclination, we perform a bootstrap in which 222 randomly chosen sites from the original data set are analyzed in the same fashion. Results from twenty such bootstrapped data sets are shown as thin lines in Plate 2c. A histogram of 1000 crossing points of bootstrap curves with the model elongation-inclination line are plotted in Plate $2 \mathrm{~d}$. The mode of the bootstrapped crossings is at an inclination of $63^{\circ}$ with $95 \%$ of the crossings falling between $56^{\circ}$ and $69^{\circ}$. Other paleosecular variation 
models (e.g., CJ98.GAD) will give different results in detail. However, the estimates are all within a few degrees of each other because the largest differences among models occur in the low inclination regions and all are unity at the pole. The region most sensitive to inclination error is at inclinations of near $45^{\circ}$ where the various models are relatively consistent.

The results of the elongation-inclination method virtually rule out a significant role for axial octupolar fields as the cause for the inclination bias observed in the Asian sedimentary rocks and strongly support the sedimentary flattening hypothesis of Gilder et al. [2001], Tan et al. [2003] and Gilder et al. [2003].

\section{CONCLUSIONS}

We have created a simple statistical field model based on the Giant Gaussian Process approach pioneered by Constable and Parker [1988]. The model was designed to fit currently available estimates for average field intensity and VGP scatter as functions of latitude while retaining the elegant simplicity of the Constable-Parker model. Our model fits the average field intensity found by Selkin and Tauxe [2000] and the VGP scatter as a function of latitude of McElhinny and McFadden [1997]. Realizations of the TK03.GAD model lead to the following observations:

1. Our model fits the paleomagnetic data quite well; it suggests however, that the Lowes spectrum of the present field is at the upper bounds of behavior for the geomagnetic field.

2. In general, directions representing paleosecular variation of the geomagnetic field are not expected to be Fisher distributed, while VGP distributions resulting from those directional data sets are likely to be at least circularly symmetric (although not, in fact, Fisher distributed). The direction of elongation in GAD fields is North-South with the maximum elongation at the equator. Statistical treatment of directional data sets should use a bootstrap technique that assumes no $a$ priori distribution. Furthermore, mapping of circularly symmetric VGP distributions results in elliptical directional distributions with a shallow bias in the mean inclination with respect to the expected direction at mid- latitude sites.

3. Recent PSV models are based on data sets that have attempted to eliminate transitional directions from the analysis of distributions of directions and VGPs by using various VGP colatitude cutoff angles. Our statistical field model has no reversals built into it (in fact the $2_{1}{ }^{0}$ term changed sign only 26 times in 10,000 simulations), yet has many VGPs that exceed these arbitrary cutoffs, particularly from high latitude sites of observation. The resulting statistical parameters (e.g., VGP scatter) will therefore underestimate the true variability of the non-transitional geomagnetic field.
4. While large deviations from the geocentric axial dipole axis are always associated with low intensities, low intensities are not always associated with deviant field directions, especially for low latitude sites of observation. Hence "excursions", which are by definition large deviations in direction, cannot be reliably identified by low paleointensity values alone and will only rarely be observed globally.

The principal advantage of using a statistical paleosecular variation model is that we can evaluate various processes that have been called upon to explain anomalous inclinations observed in several data sets of late. In particular, we have varied the contribution of the axial octupolar gauss coefficient and evaluated its effect on the distribution of directions generated from that particular field model. We compared realizations of the octupolar field model with the distribution of directions derived from our TK03.GAD model after "flattening" using the well known inclination error formula of King [1955] $\left[\tan \left(I_{o}\right)=f \tan \left(I_{f}\right)\right]$ where $f$ is the "flattening factor". Our analyses suggest the following:

1. The contribution of non-zero non-GAD terms to the geomagnetic field changes the distribution of directions. The contribution of a non-zero average axial octupole of the same sign as the axial dipole enhances $\mathrm{N}-\mathrm{S}$ elongation of the observed directions as well as creating a shallow bias. The predicted distributions are distinctly different from those expected from sedimentary inclination error, which are elongate East-West.

2. We develop two procedures for "correcting" inclinations that have suffered from sedimentary flattening. The first is the correction-by-site (CBS) method. The CBS method requires no a priori assumption about the distribution of paleofield directions. It relies instead on the assumption that at a site level, variations in direction are largely due to random errors during sampling and measurement; these are routinely expected to result in Fisher distributed data. If a sufficient number of samples ( 20) are available for each site, the value of $f$ can be found that minimizes elongation, returning the data to their original (by assumption) circularly symmetric state. Site means from these adjusted directions can then be used to calculate the mean direction of the entire study. We stress that the sampling strategy must be designed to sample an instant in time and not average out secular variation. Furthermore, each site must sample a homogeneous lithology to ensure a uniform value of $f$ for all samples from the same site.

3. A second method of inclination error correction relies on the behavior of the elongation versus inclination of the statistical field model TK03.GAD which has the best-fitting polynomial function of $E=2.88-0.0087 I-0.005 I^{2}$. Directions are inverted with a range of values of the flattening fac- 
tor using the equation $\tan \left(I^{* *}\right)=(1 / f) \tan \left(I_{o}\right)$ where $I_{o}$ is the observed inclination, $I^{* *}$ is the transformed inclination and $f$ is the assumed flattening factor. Elongation and mean inclination are calculated for each set of transformed data and plotted in an elongation-inclination plot. The inclination at which the transformed data cross the model is the inclination/elongation pair consistent with the field model. 95\% confidence bounds can be found using a bootstrap.

4. Performing the elongation/inclination procedure on the large Oligo-Miocene data set of Gilder et al. [2001] results in an estimate of $63_{56}{ }^{69}$ for the inclination, precisely that predicted from the apparent polar wander path for Eurasia of Besse and Courtillot [2002]. The initial distribution of data is elongate $\mathrm{E}-\mathrm{W}$, which precludes an axial octupolar field as the cause of the inclination anomaly. Depositional inclination error is therefore the likely cause for inclination bias in the Asian red beds.

5. We suspect that inclination error is prevalent in ancient redbeds that carry a detrital magnetization. This will contribute to a shallow bias in statistical distribution of inclinations, as has been observed in pre-Cenozoic data (e.g., Kent and Smethurst [1998]). The ability to diagnose sedimentary inclination error by the methods described here should be strong motivation for adequate sampling and for reporting results at the sample level. The fact that data from crystalline rocks may also show a shallow bias (e.g., Kent and Smethurst [1998]) could mean that these crystalline data may suffer from some other artifacts, such as undetected tilting. In the meantime, paleopoles for tectonic plates based on sedimentary data, particularly with detrital hematite as the carrier of remanence, should be used with caution.

Acknowledgments. We thank Rob Van der Voo, Wout Krijgsman, Yves Gallet, and Vincent Courtillot for stimulating conversations. Ken Kodama and Stuart Gilder provided helpful reviews and Cathy Constable and Catherine Johnson significantly improved the manuscript with thoughtful suggestions. We thank Julie Bowles for careful proof reading. Stuart Gilder kindly sent us his data. We are grateful to Daniel Staudigel who wrote the program "CloudView" which projects paleomagnetic vectors in three dimensional, color coded plots. This work was partially supported by NSF Grant EAR0003395. Lamont-Doherty Earth Observatory contribution \#6551.

\section{REFERENCES}

Baag, C., and C. Helsley, Shape analysis of paleosecular variation data, J. Geophys. Res, , 4923-4932, 1974.

Bazhenov, M., and A. Mikolaichuk, Paleomagnetism of Paleogene basalts from the Tien Shan Kyrgyzstan: rigid Eurasia and dipole geomagnetic field, Earth Planet. Sci. Lett., , 155-166, 2002.

Beck, M., On the shape of paleomagnetic data sets, J. Geophys. Res., 104, 25,427-25,441, 1999.
Besse, J., and V. Courtillot, Apparent and true polar wander and the geometry of the geomagnetic field over the last $200 \mathrm{Myr}, \mathrm{J}$. Geophys. Res, , doi:10.1029/2000JB000,050, 2002.

Bingham, C., Distributions on the sphere and on the projective plane, Ph.d. thesis, Yale University, 1964.

Bingham, C., An antipodally symmetric distribution on the sphere, Ann. Statist., 2, 1201-1225, 1974.

Carlut, J., and V. Courtillot, How complex is the time-averaged geomagnetic field over the past 5 Myr?, Geophys. J. Int.,134, 527-544, 1998.

Carlut, J., X. Quidelleur, V. Courtillot, and G. Boudon, Paleomagnetic directions and $\mathrm{K} / \mathrm{Ar}$ dating of 0 to $1 \mathrm{Ma}$ lava flows from La Guadeloupe Island (French West Indies): Implications for time-averaged field models, J. Geophys. Res., 105, 835-849, 2000.

Chauvin, A., H. Perroud, and M. Bazhenov, Anomalous low paleomagnetic inclinations from Oligocene-Lower Miocene red beds of the south-west Tien Shan, Central Asia, Geophys. J. Int., 126, 303-313, 1996.

Cogné, J., N. Halim, Y. Chen, and V. Courtillot, Resolving the problem of shallow magnetizations of Tertiary age in Asia: insights from paleomagnetic data from the Qiangtang, Kunlun, and Qaidam blocks (Tibet, China), and a new hypothesis, J. Geophys. Res, 104, 17,715-17,734, 1999.

Constable, C., and C. Johnson, Anisotropic paleosecular variation models: implications for geomagnetic field observables, Phys. Earth Planet. Int., 104, 35-51, 1999.

Constable, C., and R. L. Parker, Statistics of the geomagnetic secular variation for the past 5 m.y., J. Geophys. Res., 115, 11,569-11,581, 1988.

Cox, A., Analysis of present geomagnetic field for comparison with paleomagnetic results, J. Geomag. Geoelectr., 13, 101-112, 1962.

Cox, A., Research note: Confidence limits for the precision parameter, K, Geophys. J. Roy. Astron. Soc, 17, 545-549, 1969.

Creer, K., E. Irving, and Nairn, Paleomagnetism of the Great Whin Sill, Geophys. J. Int., 17, 306-323, 1959.

Creer, K. M., Computer synthesis of geomagnetic paleosecular variations, Nature, 2, 695-699, 1983.

Dupont-Nivet, G., Z. Guo, R. Butler, and C. Jia, Discordant paleomagnetic direction in Miocene rocks from the central Tarim Basin: evidence for local deformation and inclination shallowing, Earth Planet. Sci. Lett., 199, 473-482, 2002.

Fisher, R. A., Dispersion on a sphere, Proc. Roy. Soc. London, Ser. A, 217, 295-305, 1953.

Gilder, S., Y. Chen, and S. Sen, Oligo-Miocene magnetostratigraphy and rock magnetism of the Xishuigou section, Subei (Gansu Province, western China) and implications for shallow inclinations in central Asia, J. Geophys. Res, 106, 30,505-30,521, 2001.

Gilder, S., Y. Chen, J. Cogné, X. Tan, V. Courtillot, D. Sun, and Y. Li, Paleomagnetism of Upper Jurassic to Lower Cretaceous volcanic and sedimentary rocks from the western Tarim Basin and implications for inclination shallowing and absolute dating of the M-O (ISEA?) chron, Earth Planet. Sci. Lett., 206, 587-600, 2003.

Gubbins, D., and P. Kelly, Persistent patterns in the geomagnetic field over the past 2.5 Myr, Nature, 365, 829-832, 1993. 
Hoffman, K., A method for the display and analysis of transitional paleomagnetic data, J. Geophys. Res., 89, 6285-6292, 1984.

Irving, E., Paleomagnetism and Its Application to Geological and Geophysical Problems, John Wiley and Sons, Inc., 1964.

Johnson, C., and C. Constable, The time averaged geomagneitc field: global and regional biases for 0-5 Ma, Geophys. J. Int., 131, 643-666, 1997.

Johnson, C. L., and C. G. Constable, The time-averaged geomagnetic field as recorded by lava flows over the last $5 \mathrm{Myr}$, Geophys. J. Int., 122, 489-519, 1995.

Johnson, C. L., and C. G. Constable, Palaeosecular variation recorded by lava flows over the past five million years, Phil. Trans. R. Soc. Lond. A., 354, 89-141, 1996.

Johnson, C. L., and C. G. Constable, Persistently anomalous Pacific geomagnetic fields, Geophys. Res. Lett., 25, 1011-1014, 1998.

Kelly, P., and D. Gubbins, The geomagnetic field over the past 5 million years, Geophys. J. Int., 128, 315-330, 1997.

Kent, D. V., and M. Smethurst, Shallow bias of paleomagnetic inclinations in the Paleozoic and Precambrian, Earth Planet. Sci. Lett., 160, 391-402, 1998.

King, R. F., The remanent magnetism of artificially deposited sediments, Mon. Nat. Roy. astr. Soc., Geophys. Suppl., 7, 115-134, 1955.

Kono, M., Distributions of paleomagnetic directions and poles, Phys. Earth Planet. Int., 103, 313-327, 1997.

Kono, M., and H. Tanaka, Mapping the Gauss coefficients to the pole and the models of paleosecular variation, J. Geomag. Geoelectr., 47, 115-130, 1995.

Love, J., and C. G. Constable, Gaussian statistics for paleomagnetic vectors, Geophys. J. Int., 152, 515-565, 2003.

Lowes, F., Spatial power spectum of the main geomagnetic field and extrapolation to the core, Geophys. J. R. Astron. Soc., 36, 717-730, 1974.

McElhinny, M. W., and P. L. McFadden, Palaeosecular variation over the past $5 \mathrm{Myr}$ based on a new generalized database, Geophys. $J$. Int., 131, 240-252, 1997.

McElhinny, M. W., P. L. McFadden, and R. T. Merrill, The timeaveraged paleomagnetic field 0-5 Ma, J. Geophys. Res., 101, 25,007-25,027, 1996.

McFadden, P. L., R. T. Merrill, and M. W. McElhinny, Dipole/Quadrupole family modeling of paleosecular variation, J. Geophys. Res, 93, 11,583-11,588, 1988.

Merrill, R. T., and M. W. McElhinny, Anomalies in the time-averaged paleomagnetic field and their implications for the lower mantle, Rev. Geophys. Space Phys., 15, 309-322, 1977.

Opdyke, N. D., and K. W. Henry, A test of the Dipole Hypothesis, Earth Planet. Sci. Lett., 6, 139-151, 1969.

Quidelleur, X., J. P. Valet, V. Courtillo, and G. Hulot, Long-term geometry of the geomagnetic field for the last five million years: An updated secular variation database, Geophys. Res. Lett., 21, 1639-1642, 1994.

Scheidegger, A. E., On the statistics of the orientation of bedding planes, grain axes, and similar sedimentological data, U.S. Geo. Surv. Prof. Pap., 525-C, 164-167, 1965.
Schneider, D., and D. V. Kent, The time-averaged paleomagnetic field, Rev. Geophys., 18, 71-96, 1990.

Schneider, D. A., and D. V. Kent, The paleomagnetic field from equatorial deep-sea sediments: axial symmetry and polarity asymmetry, Science, 242, 252-256, 1988.

Selkin, P., and L. Tauxe, Long-term variations in paleointensity, Phil. Trans. Roy. Astron. Soc., 358, 1065-1088, 2000.

$\mathrm{Si}$, J., and R. Van der Voo, Too-low magnetic inclinations in central Asia: an indication of a long-term Tertiary non-dipole field?, Terra Nova, 13, 471-478, 2001.

Tan, X., K. Kodama, H. Chen, D. Fang, D. Sun, and Y. Li, Paleomagnetism and magnetic anisotropy of Cretaceous red beds from the Tarim basin northwest China: Evidence for a rock magnetic cause of anomalously shallow paleomagnetic inclinations from central Asia, J. Geophys. Res, 108, doi:10.1029/2001JB001,608, 2003.

Tanaka, H., Circular asymmetry of the paleomagnetic directions observed at low latitude volcanic sites, Earth Planets Space, 51, 1279-1286, 1999.

Tanaka, H., M. Kono, and H. Uchimura, Some global features of paleointensity in geological time, Geophys. J. Int., 120, 97-102, 1995.

Tauxe, L., Paleomagnetic Principles and Practice, Kluwer Academic Publishers, 1998.

Tauxe, L., and D. V. Kent, Properties of a detrital remanence carried by hematite from study of modern river deposits and laboratory redeposition experiments, Geophys. Jour. Roy. astr. Soc., 77, 543-561, 1984.

Tauxe, L., C. Constable, C. Johnson, W. Miller, and H. Staudigel, Paleomagnetism of the Southwestern U.S.A. recorded by 0-5 Ma igneous rocks, Geochem., Geophys., Geosyst., DOI 10.1029/2002GC000343, 2003.

Thomas, J.-C., H. Perroud, P. Cobbold, M. Bazhenov, V. Burtman, A. Chauvin, and E. Sdybakasov, A paleomagnetic study of Tertiary formations from the Kyrgys Tien-Shan and its tectonic implications, J. Geophys. Res, 98, 9571-9589, 1993.

Vandamme, D., A new method to determine paleosecular variation, Phys. Earth Planet. Int., 85, 131-142, 1994.

Van der Voo, R., and T. H. Torsvik, Evidence for late Paleozoic and Mesozoic non-dipole fields provides an explanation for the Pangea reconstruction problems, Earth Planet Sci Lett, 187, 71-81, 2001.

Westphal, M., Did a large departure from the geocentric axial dipole hypothesis occur during the Eocene? Evidence from the magnetic polar wander path of Eurasia, Earth Planet. Sci. Lett., 117, 15-28, 1993.

L. Tauxe, Scripps Institution of Oceanography, La Jolla, California 92093-0220. (1tauxe@ucsd.edu)

Dennis V. Kent, Department of Geological Sciences, Rutgers University, Piscatoaway, New Jersey 08854 \& Lamont Doherty Geological Observatory, Palisades, New York 10964. (dvk@1deo.columbia.edu) 